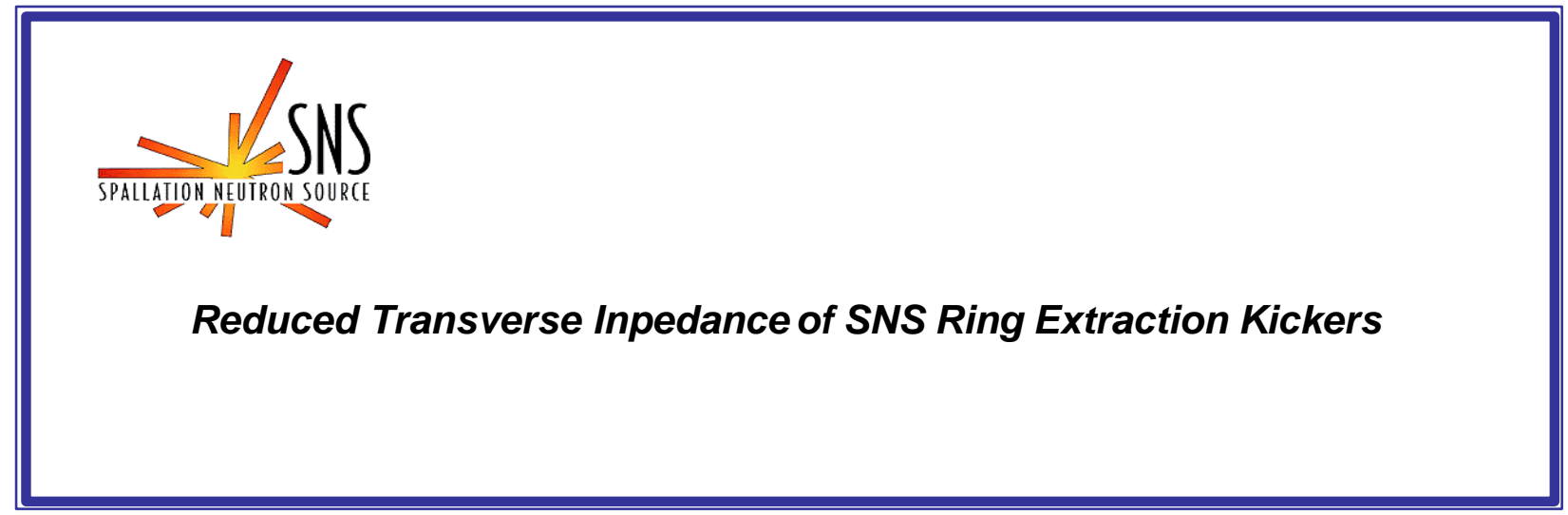

BNL/SNS TECHNICAL NOTE

NO. 112

Revision A

D. Davino, Jian-lin Mi, and N. Tsoupas

BNL, Upton, NY 11973, USA

August 27, 2002

COLLIDER-ACCELERATOR DEPARTMENT BROOKHAVEN NATIONAL LABORATORY UPTON, NEW YORK 11973 


\title{
Reduced transverse impedance of SNS ring extraction kickers
}

\author{
D. Davino, Jian-lin Mi and N. Tsoupas \\ Brookhaven National Laboratory, Upton, NY 11973 USA
}

\begin{abstract}
In this report it is presented a way to reduce the transverse impedance contribution of the fourteen extraction kickers for the SNS accumulator ring. This is done by increasing the vertical aperture of the kickers, namely the equivalent pole width. The gap distances between the pole surfaces are almost untouched instead. With respect to the old dimensions, almost a factor two of reduction is achieved for the transverse impedance. The total contribution of fourteen kickers below $10 \mathrm{MHz}$, with $25 \Omega$ termination, is now:

$$
\mathrm{Z}_{\mathrm{y}}=(33.2+\mathrm{j} 125) \mathrm{k} \Omega / \mathrm{m}
$$
\end{abstract}

A summary of the model used for simulations and fitting criteria are also given.

\section{Introduction}

The transverse coupling impedance of a circular accelerator or storage ring can cause beam instabilities and must be carefully monitored in order to keep its value within the impedance budget. The success of the SNS (Spallation Neutron Source) will largely depend on reducing the transverse coupling impedance of the accumulator ring. Next to the resistive wall impedance, the biggest contribution is made by the ferrite kicker magnets. Thus, an extensive experimental and theoretical program was carried out to achieve this design goal [1,2,3].

The extraction system of the SNS accumulator ring has 14 kickers and the apertures of those kickers were adjusted with the betatron function to yield the overall SNS ring acceptance [4].
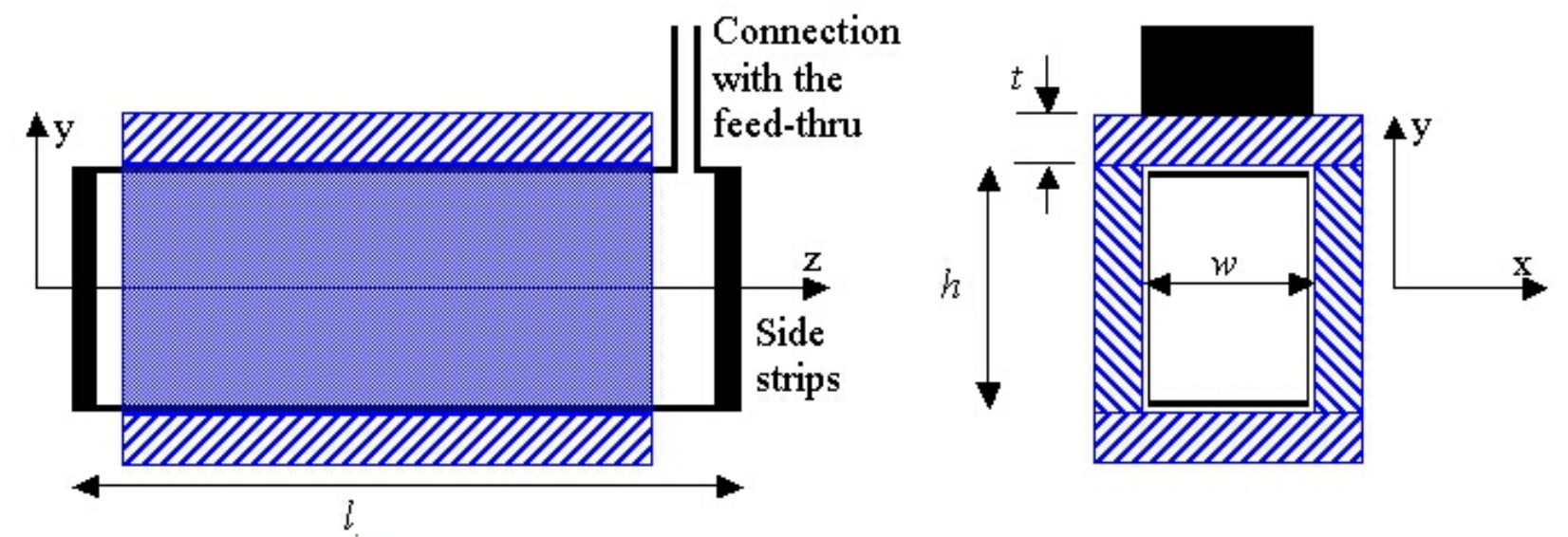

Fig. 1. Schematic view of SNS window frame extraction kicker. Blue frames represent ferrite blocks. " $z$ " is the beam axis. $h$ is the aperture height, $w$ is the width, $l$ is the bus-bar length and $t$ is the ferrite thickness. 
A prototype of one of the fourteen SNS extraction kickers was made and measured with the twinwire technique [5]. A schematic view of that kicker is shown in Fig. 1. The kicker dimensions are: $h=24.8 \mathrm{~cm}, w=15.9 \mathrm{~cm}, l=36 \mathrm{~cm}$ and $t=2.54 \mathrm{~cm}$. The ferrite is CMD5005 type [6] and the two bus-bar plates are connected through side strips. A half-size bus-bar, $h=12.4 \mathrm{~cm}$, was also made; in the following we refer to it as half-size kicker. The connections to the feed-thru are made by a prolongation of the bus-bar. In order to damp the natural kicker resonance, a $25 \Omega$ power resistor is placed in parallel with the external circuit.

In the next section, the measurements of the full and half-size kickers, and a scaling law for the transverse impedances, are presented. In the third section, the new dimensions of the kickers are presented and commented and in the last section, equivalent formulae of the transverse impedance are introduced.

\section{Scaling law}

The analysis of the bench measurements performed on the SNS extraction kicker prototype [1,2] led to an equivalent circuit for ferrite lumped kickers and some important properties of those structures were derived from it [7]. One of the most interesting is the scaling law with kicker height, $h$, of the contribution to the impedance, coupled to the external circuit:

$$
Z_{y 2}=Z_{y 1} \cdot\left(\frac{h_{1}}{h_{2}}\right)^{2}
$$

This law can be considered as an upper limit for the impedances. Indeed, it is neglected the fact that the aperture height changes the magnet inductance too and therefore the resonant peak frequency.

For the uncoupled contribution to the impedance, here identified with the measurement with shorted bus-bar, one can consider a scaling law that goes, in first approximation, with the square of the aperture gaps, $w$, [7] namely:

$$
Z_{y 2}^{s h}=Z_{y 1}^{s h} \cdot\left(\frac{w_{1}}{w_{2}}\right)^{2}
$$

In order to verify the eq. (1), a half-size model was assembled and measured with the existing ferrite bricks and a new simplified bus-bar. The results are shown in Fig. 2 where the measured impedances of the full and half-size kickers are compared with the prediction of the equivalent model in [7].

The resonant frequency of the half size model is about $\sqrt{2}$ times the full one, because of the halved magnet inductance. With open termination, the peak of the measured impedance is in very good agreement with the application of the equivalent model for the kicker that gives a factor $4 / \sqrt{2}$. The $25 \Omega$ termination case also shows a good agreement with the application of the model. However, it is apparent that the scaling law (1) represents a reasonable upper limit to estimate the 
contribution to the impedance budget of kickers with reduced aperture with respect to the measured one.
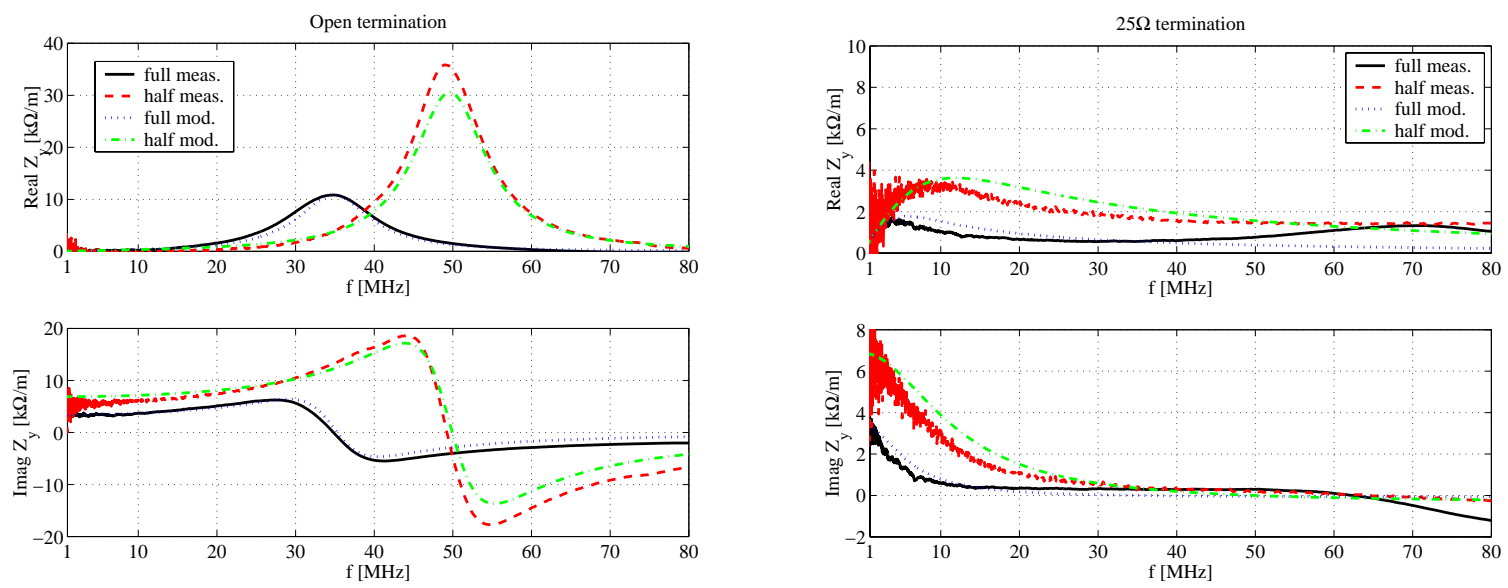

Fig. 2. Transverse impedances with open termination at the bus-bar port (left) and $25 \Omega$ (right). The measurements curves (black and red) are compared with the predicted model curves (blue and green).

Another important aspect of the scaling law is that it shows a way to reduce the transverse impedance contribution of the extraction system: by taking into account the other constrains (voltage, current, rise time, etc.) to the kickers geometry ( $h$ and $w$ ), one can reduce the total transverse impedance by maximizing the kickers height, $h$.

\section{Electrical aspects and new kickers dimensions}

Fourteen magnets will be individually energized by fourteen BPFN type (Blumlein Pulse Forming Network) high voltage modulators. There are two $50 \Omega$ coaxial cables connected in parallel to transfer the current pulse from BPFN to the ferrite magnet. Then, the cables are matched with the $25 \Omega$ resistor connected at the cable BPFN side. We chose BPFN operation voltage at $35 \mathrm{kV}$, in order to increase the system operation reliability and to be optimal at the PFN cost. According to the simulation and the prototype tests, the magnet operating current pulse will be less than $2.5 \mathrm{kA}$ while the BPFN operating at $35 \mathrm{kV}$. At this current pulse, the magnetic field B is estimated to be from 142 to 251 Gauss, depending on the magnet aperture horizontal dimensions. More details about this subject can be found in [8].

The proton beam extraction function should move the beam from the accumulation ring to the beam transfer line completely and cleanly. So, it is necessary to establish the extraction magnet field during the $250 \mathrm{nS}$ beam gap. The current pulse rise time from 1 to $95 \%$ is set at around $200 \mathrm{nS}$ to guarantee the beam is kicked from the accumulate ring to beam transfer line totally.

In order to reduce the magnet pulse current rise time, the magnet inductance has to be limited to meet the BPFN impedance and the magnet current pulse requirement. The magnet inductance mainly relies on the magnet dimensions and magnet feedthrough. The total magnet and feedthrough inductance is limited at $1.1 \mu \mathrm{H}$ to meet the pulse rise time constraint. 
With respect to the previous arguments, the ferrite magnet dimensions can be re-estimated. The main modifications are at the first seven magnets where the vertical dimensions are increased. The new ferrite magnet dimensions are listed in the following table. From the table, the magnet operating currents are still limited below $2.5 \mathrm{kA}$.

\begin{tabular}{|c|c|c|c|c|c|c|c|}
\hline kicker & Horiz. $\boldsymbol{w}(\mathbf{m})$ & Vert. $\boldsymbol{h}(\mathbf{m})$ & Length $\boldsymbol{l}(\mathbf{m})$ & Kick $(\mathbf{m r a d})$ & $\mathbf{I}(\mathbf{k A})$ & $\mathbf{B} / \mathbf{s e c t}(\mathbf{G a u s s})$ & $\begin{array}{c}\mathbf{L}=\boldsymbol{\mu}_{\boldsymbol{0}} \boldsymbol{h} \boldsymbol{l} / \boldsymbol{w} \\
(\boldsymbol{\mu} \mathbf{H})\end{array}$ \\
\hline $\mathrm{K} 11$ & 0.120 & 0.166 & 0.40 & 1.78 & 2.40 & 251 & 0.695 \\
\hline $\mathrm{K} 12$ & 0.145 & 0.200 & 0.40 & 1.47 & 2.39 & 208 & 0.693 \\
\hline $\mathrm{K} 13$ & 0.145 & 0.200 & 0.40 & 1.47 & 2.39 & 208 & 0.693 \\
\hline $\mathrm{K} 14$ & 0.178 & 0.195 & 0.51 & 1.52 & 2.40 & 170 & 0.702 \\
\hline $\mathrm{K} 15$ & 0.178 & 0.195 & 0.51 & 1.52 & 2.40 & 170 & 0.702 \\
\hline $\mathrm{K} 16$ & 0.211 & 0.233 & 0.51 & 1.28 & 2.40 & 143 & 0.708 \\
\hline $\mathrm{K} 17$ & 0.211 & 0.233 & 0.51 & 1.28 & 2.40 & 143 & 0.708 \\
\hline $\mathrm{K} 21$ & 0.162 & 0.233 & 0.43 & 1.43 & 2.44 & 189 & 0.777 \\
\hline $\mathrm{K} 22$ & 0.162 & 0.233 & 0.43 & 1.43 & 2.44 & 189 & 0.777 \\
\hline $\mathrm{K} 23$ & 0.162 & 0.233 & 0.43 & 1.43 & 2.44 & 189 & 0.777 \\
\hline $\mathrm{K} 24$ & 0.162 & 0.233 & 0.43 & 1.43 & 2.44 & 189 & 0.777 \\
\hline $\mathrm{K} 25$ & 0.151 & 0.243 & 0.39 & 1.40 & 2.44 & 203 & 0.789 \\
\hline K26 & 0.151 & 0.243 & 0.39 & 1.40 & 2.44 & 203 & 0.789 \\
\hline K27 & 0.151 & 0.243 & 0.39 & 1.40 & 2.44 & 203 & 0.789 \\
\hline
\end{tabular}

\section{Impedance contribution and equivalent models for simulations}

\section{Contribution to the impedance budget}

By using the scaling law (1), in the SNS note n. 102, the impedance contribution of all the fourteen kickers with transverse aperture able to accommodate the beam size, was calculated to be 32.2 times the one of the measured kicker [2], leading to:

$$
\begin{array}{lll}
Z_{y}=(74+j 209) \mathrm{k} \Omega / m & \text { for } & f<10 \mathrm{MHz}, \\
Z_{y}=(45+j 164) \mathrm{k} \Omega / \mathrm{m} & \text { for } & \mathrm{f} \sim 50 \mathrm{MHz} .
\end{array}
$$

With increased vertical apertures of previous table, the application of scaling law (1) gives a factor 18.2 for the coupled contribution of the fourteen kickers.

Moreover, the results of the measurements of the transverse coupling impedance were interpreted again with more reliable values about the characteristic impedance of the twin-wire line, $260 \Omega$, and the wire spacing, $45.6 \mathrm{~mm}$. However, in view of the various measuring errors, all the quoted transverse impedances should be considered within $20 \%$ accuracy.

A safe evaluation of the impedance below $10 \mathrm{MHz}$ with a $25 \Omega$ resistive termination leads to a peak of $(1.8+\mathrm{j} 6.5) \mathrm{k} \Omega / \mathrm{m}$. Then, the updated contribution of all the 14 kickers (with the vertical apertures given in the previous table) is:

$$
\begin{array}{lll}
\mathrm{Z}_{\mathrm{y}}=18.2 \cdot(1.8+\mathrm{j} 6.5) \mathrm{k} \Omega / \mathrm{m}=(33.2+\mathrm{j} 125) \mathrm{k} \Omega / \mathrm{m} & \text { for } & \mathrm{f}<10 \mathrm{MHz} \\
\mathrm{Z}_{\mathrm{y}}=(12+\mathrm{j} 62) \mathrm{k} \Omega / \mathrm{m} & \text { for } & \mathrm{f} \sim 50 \mathrm{MHz}
\end{array}
$$




\section{Measurement models for one kicker}

The transverse impedance of a ferrite lumped kicker in the kick direction has two contributions: one is coupled with the external circuit and another one not. The simplest way to represent the first contribution is by means of a RLC resonator model:

$$
Z_{y}^{\text {open }}=\frac{c}{\omega h^{2}}[1 / R+1 /(j \omega L)+j \omega C]^{-1}
$$

where $R=490 \Omega, L=0.7 \mu \mathrm{H}, C=28.7 \mathrm{pF}$ and $h=0.248 \mathrm{~m}$ for the measured prototype with open termination. The resistance $\mathrm{R}$ represents the ferrite losses. The external circuit, downstream of the kicker, must be added in parallel in order to have the impedance in operational conditions. The PFN feed-thru is well approximated by a shunt capacitance of about $20.5 \mathrm{pF}$. Finally, the $25 \Omega$ matching resistor is added in parallel, leading to:

$$
Z_{y}^{F T / 25 \Omega}=\frac{c}{\omega h^{2}}[1 / 23.9+1 /(j \omega 0.7 \mu)+j \omega 49.2 p]^{-1}
$$

This formula can be written in equivalent way as [9]:

$$
Z_{y}^{F T / 25 \Omega}=\frac{c}{\omega} R_{s}\left[1+j Q\left(f_{r} / f-f / f_{r}\right)\right]^{-1},
$$

where $f_{r}=27.1 \mathrm{MHz}, Q=0.199, R_{s}=387 \Omega / \mathrm{m}^{2}$. It is worth noting that eq. 4 seems not to fit very well the measurement with $25 \Omega$ termination and feed-thru. This is due to the poor quality of the measurements at low frequencies (below $10 \mathrm{MHz}$ ) and to the uncertainty of the connections for the load. On the other hand, the fitting of the opened kicker case is very reliable because of the resonance, and the numerical scaling to the actual loads is based on a well-tested model [7] and can be considered reliable too.

About the uncoupled contribution to the transverse impedance, hereby identified with the measurement with shorted bus-bar, it can be modeled with a lossy inductor and the losses are proportional with the frequency, giving $1 \mathrm{k} \Omega / \mathrm{m}$ at $100 \mathrm{MHz}$. For instability codes purposes, it is still possible to model this contribution with a factitious resonator:

$$
Z_{y}^{s h}=\frac{c}{\omega} R_{s}^{s h}\left[1+j Q^{s h}\left(f_{r}^{s h} / f-f / f_{r}^{s h}\right)\right]^{-1}
$$

where $f_{r}^{s h}=400 \mathrm{MHz}, Q^{s h}=1.325, R_{s}^{s h}=50 \mathrm{k} \Omega / \mathrm{m}^{2}$. In the frequency range of interest, this resonator gives an almost-constant imaginary part of $4.5 \mathrm{k} \Omega / \mathrm{m}$ and a real part, linear with frequency, of $0.9 \mathrm{k} \Omega / \mathrm{m}$ at $100 \mathrm{MHz}$ in good agreement with recent measurements on magnets [7]. 


\section{Application of the scaling laws}

Following the same arguments of the previous paragraphs, the fitting curve of the total of 14 kickers can be obtained by considering 18.2 times (scaling factor) the one represented by the eq. (4). But, it is worth noting that the changes of the aperture dimensions do change the resonator parameters too. For instance, the inductance is in first approximation given by $L=\mu_{0} h l / w$, and then, by assuming the same capacitance (mainly given by the termination to the feed-thru) and losses for all the kickers, one obtains 14 resonators with slightly different resonant frequency. Fig. 4 shows all the transverse impedance curves of them.

In Fig. 5, the sum of previous curves is compared with the 18.2 times scaled contribution of one kicker. The approximation by considering only one resonator is relatively small, and for the sake of simplicity, one can consider one resonator model in numerical beam-instability codes.
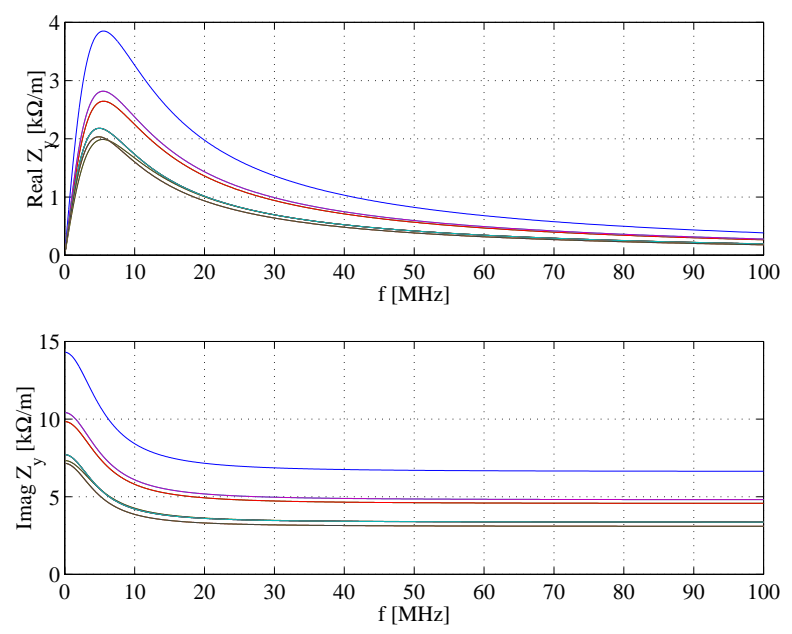

Fig. 4. The transverse impedances of all the 14 kickers with aperture-scaled inductances.
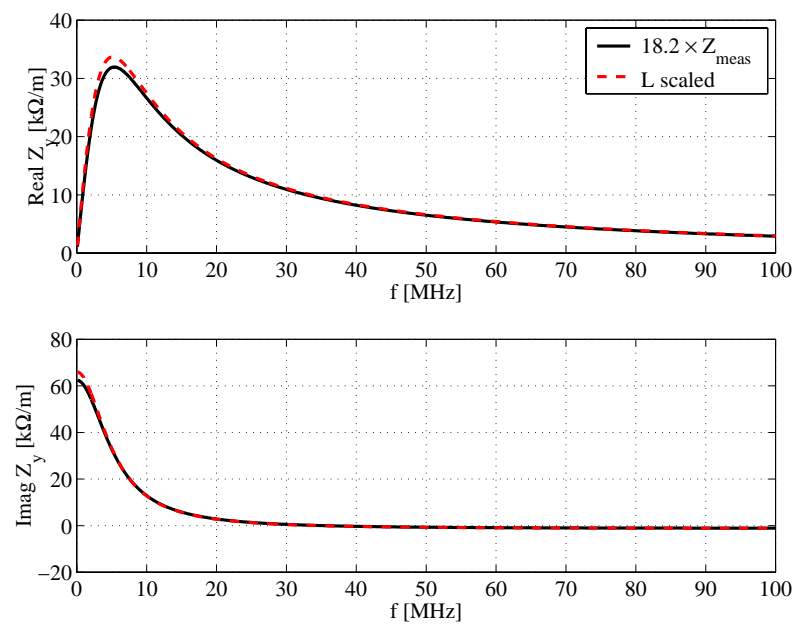

Fig. 5. The sum of the transverse impedance of the 14 kickers with aperture-scaled inductances

( $\mathrm{L}$ scaled curve) and the scaled measured impedances $\left(18.2 \times \mathrm{Z}_{\text {meas }}\right.$ curve). 
With regards to the uncoupled contribution, the application of eq. (2) to the SNS kickers and the geometry of measured kicker, gives about a factor 14 that is the number of kickers too. Therefore, the eq. (5), representing the short circuit measurement, should be multiplied by a factor 14. Also, one should not use the eq. (1) to scale the uncoupled contribution.

Finally, the total impedance of 14 SNS kickers can be modeled as:

$$
\begin{aligned}
Z_{y}^{\text {TOT }=} & \frac{c}{\omega} 7043[1+j 0.199(27.1 / f-f / 27.1)]^{-1}+ \\
& \frac{c}{\omega} 700 \cdot 10^{3}[1+j 1.325(400 / f-f / 400)]^{-1}
\end{aligned}
$$

with frequencies in $\mathrm{MHz}$.

\section{Acknowledgements}

The authors would like to thank Drs. H. Hahn, A. Fedotov and J. Wei for fruitful discussions.

\section{References}

[1] D. Davino and H. Hahn, Transverse impedance measurement of a simplified model of the SNS extraction kicker, BNL/SNS No. 088, BNL, March 2001.

[2] D. Davino and H. Hahn, Measurements and analysis of the transverse coupling impedance of the SNS extraction kickers, BNL/SNS No. 102, BNL, October 2001.

[3] H. Hahn and D. Davino, SNS extraction kicker with low- $\mu$ ferrite, BNL/SNS No. 109, BNL, June 2002.

[4] N. Tsoupas et al., Beam extraction from the SNS ring and design of the extraction kickers, Proc. EPAC 2000, Vienna, p. 2270.

[5] F. Caspers, in Handbook of Accelerator Physics and Engineering, ed. A.W. Chao and M. Tigner, (World Scientific, Singapore, 1998), p. 571.

[6] Ceramic Magnetics inc., Fairfield, NJ, USA.

[7] D. Davino and H. Hahn, submitted to Physical Review ST-AB, August 2002.

[8] J. Mi, C. Pai, D. Davino, H. Hahn, R. Lambiase, Y.Y. Lee, W. Meng, J. Sandberg, N. Tsoupas, W. Zhang, D. Warburton, SNS extraction kicker system and first article PFN test, Proc. EPAC 2002, Paris.

[9] A.W. Chao, in Physics of collective beam instabilities in high energy accelerators, ed. Mel Month, (John Wiley \& Sons, Inc., New York, 1993), p. 75. 